\title{
Panorama das hierarquias no campo da educação de deficientes auditivos e surdos no período de 1987-2017
}

DOI: https://doi.org/10.22409/pragmatizes.v12i22.51444

\section{Carla Cazelato Ferrari ${ }^{1}$}

Resumo: Este artigo, por meio de balanço tendencial abrangendo o período de 1987 a 2017, teve por objetivo analisar as permanências e descontinuidades da distribuição institucional das teses e dissertações brasileiras sobre a educação de surdos, como expressão das disputas estabelecidas nesse campo, por meio dos seguintes indicadores: instância administrativa, área de conhecimento, instituição de ensino superior (IES), grau acadêmico e identificação do sujeito da pesquisa e língua prioritária.Destaca-se entre os resultados de análise a incidência de pesquisas que se desenvolveram em programas de pós-graduação da área da educação em instituições de ensino superior públicas, com investigações voltadas à escola, suas práticas e sujeitos. Assim como, observou-se a relevância de considerar, para além das questões centradas nas limitações acarretadas pela surdez, outros aspectos como condições econômicas e sociais, raça, idade e gênero, que conforme o panorama apresentado, acabaram sendo secundarizados nas investigações.

Palavras-chave: educação de surdos; pesquisa discente; hierarquias institucionais.

Panorama de las jerarquías en el campo de la educación de discapacitados auditivos y sordos en el período 1987-2017

Resumen: Este artículo, a través de un balance de tendencias que abarca el período de 1987 a 2017, tuvo como objetivo analizar las permanencias y discontinuidades en la distribución institucional de las tesis y disertaciones brasileñas sobre educación de sordos, como expresión de las disputas establecidas en este campo, a través de los siguientes indicadores : instancia administrativa, área de conocimiento, institución de educación superior (IES), título académico, identificación del sujeto de investigación y lengua prioritaria. Entre los resultados del análisis, se destaca la incidencia de investigaciones desarrolladas en programas de posgrado en el campo de la educación en instituciones públicas de educación superior, con investigaciones enfocadas en la escuela, sus prácticas y sujetos. Se observó la relevancia de considerar, además de las cuestiones centradas en las limitaciones que ocasiona la sordera, otros aspectos como las condiciones económicas y sociales, la raza, la edad y el género, aspectos que, según el panorama presentado, terminaron siendo secundarios en el panorama de las investigaciones.

Palabras clave: educación de sordos; investigación estudiantil; jerarquías institucionais

\footnotetext{
${ }^{1}$ Carla Cazelato Ferrari. Doutora em Educação. Professora da PUCSP/Pontifícia Universidade Católica de São Paulo, Brasil. E-mail: carla_cazelato@hotmail.com - https://orcid.org/0000-00024753-4742
} 
Panorama of hierarchies in the field of education of deaf and hearing impaired in the period 1987-2017

Abstract: This article, by means of a trend balance covering the period from 1987 to 2017 , aimed to analyze the regularities and discontinuities of the institutional distribution of Brazilian theses and dissertations on the education of deaf people, as an expression of the disputes established in this field, through the following indicators: administrative instance, area of knowledge, higher education institution (IES), academic degree and identification of the research subject and priority language. Among the results of the analysis, the incidence of the research developed in postgraduate programs in the area of education in public higher education institutions stands out, with investigations aimed at schools, their practices and subjects.As well, it was observed the relevance of considering, in addition to the questions centered on the limitations caused by deafness, other aspects such as economic and social conditions, race, age and gender, which according to the presented panorama, ended up being secondary in the investigations.

Keywords: deaf education; student research; institutional hierarchies

\section{Panorama das hierarquias no campo da educação de deficientes auditivos e}

\section{surdos no período de 1987-2017}

\section{Introdução}

O campo acadêmico da educação de surdos possui como uma de suas principais especificidades certas tensões, a partir de discussões concorrenciais sobre deficiência versus diferença, inclusão escolar versus escolas exclusivas, cultura diversa e plural versus cultura própria, debates que se estabeleceram no decorrer das últimas décadas e que deflagram que esse é um campo formado por perspectivas acadêmicas distintas.

Nesse sentido, este estudo tem por objetivo analisar as tendências de investigação sobre educação de surdos $^{2}$, expressas por teses e dissertações publicadas no decorrer de 1987 a 2017, em que se coteja as hierarquias constituídas em um campo específico da educação especial.

A orientação das pesquisas da educação de surdos no Brasil vem sofrendo mudanças a partir de uma vertente de estudos acadêmicos que emergiu, especialmente, a partir dos anos de 1990, junto ao movimento sociopolítico de negação da sua caracterização como deficiência auditiva e reivindicação de uma cultura própria da surdez.

\footnotetext{
${ }^{2} \mathrm{~A}$ fim de possibilitar melhor fluência na leitura do texto será utilizada a expressão "educação de surdos", englobando os estudos de pessoas com deficiência auditiva e surdez.
} 


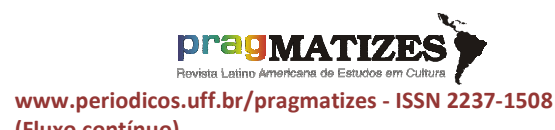

(Fluxo contínuo)
Nesse sentido, a partir da perspectiva de que a língua primeira desses sujeitos é a língua de sinais e de que os processos de oralização foram uma imposição da comunidade ouvinte sobre eles, processo esse denominado por Skliar (1998) como ouvintismo, foram produzidos inúmeros trabalhos até os anos 2000, o que passou a se denominar como vertente socioantropológica de estudos sobre a surdez, pouco tempo depois parte dessa vertente passou a ser denominada também como Estudos Surdos.

Por outro lado, desde o final do século passado, a partir da iniciativa de Bueno (1998) foram desenvolvidos estudos e investigações que se contrapuseram a perspectiva socioantropológica da surdez, na medida em que consideram a surdez como deficiência, pois a perda da audição acarreta impedimentos auditivos importantes para a vida social, assim como não constitui a única marca na composição da identidade de uma pessoa surda porque, para qualquer sujeito (surdo ou não), marcas como condição de classe, raça, gênero, local de moradia e trajetória social constituem elementos que se inscrevem na construção social da identidade dos indivíduos, valendo a pena destacar os estudos de Cukierkorn (1996, 2005), Dantas (2006), Ferrari (2010; 2017), Lima (2013), Silva (2011) e Botarelli (2014).

Nesse sentido, tomar a produção de conhecimento da educação especial, sem perder a perspectiva do âmbito da educação geral, tem fundamental relevância para se entender as lutas simbólicas travadas nesse espaço concorrencial, no caso o campo científico de uma área específica.

No campo da produção científica, as influências que possibilitam seu delineamento e redefinições são aquelas que o estruturam e definem 0 capital específico que irá regular aquilo que é eficaz e quem irá dispor de poder e autoridade. Assim, as forças que detém o poder simbólico de impor um discurso no campo definem-se relacionalmente em posições localizadas nesse espaço e pertencentes a ele, sobretudo configurando-o como um território de disputas (BOURDIEU, 2004, p.169). 


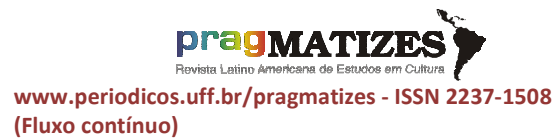

Desse modo, as teorizações de Pierre Bourdieu (1983, 2004, 2013) dão a contribuição necessária, principalmente com relação aos conceitos de campo e campo científico, entendidos a partir de um estado de relação de forças entre sujeitos ou instituições que estão engajados em um espaço de jogo. Existe, assim, o espaço de posições sociais que se retraduz em lugar de tomadas de posição, visto que são "visões tomadas a partir de um ponto, isto é, a partir de uma determinada posição no espaço social" (BOURDIEU, 1983, p.157).

Ao tomarmos as pesquisas de mestrado e doutorado como fonte de investigação sobre as tendências no campo da educação de surdos, sabese, incontestavelmente, que os programas de pós-graduação, a partir dos anos de 1970, constituíram o principal espaço de realização de pesquisas no país. Tal como, não há dúvidas de que essas investigações indicam os caminhos que as pesquisas tomaram, visto que muitas produções de artigos e livros são frutos de estudos de mestrado e doutorado.

Foram utilizadas como fonte dos dados as teses e dissertações sobre educação de surdos constantes no Catálogo da Coordenação de Aperfeiçoamento de Pessoal de Nível Superior (CAPES), compreendendo o período de 1987 a 2017, no que se refere aos dados de identificação e resumo dessas pesquisas.

A primeira parte desse levantamento foi realizado no ano de 2012 e abrangeu o período de 1987 a 2010, utilizando-se seis descritores: "surdo escola", "surdo escolarização", "surdo educação", "deficiente auditivo escola", "deficiente auditivo escolarização" e "deficiente auditivo educação", por meio da busca por "todas as palavras" e mediante supressão de duplicatas, resultando em 643 teses e dissertações sobre a educação de surdos.

A ampliação do período de coleta de dados em 7 anos, ocorreu em 2018, utilizando como procedimento a busca booleana com os mesmos descritores utilizados anteriormente, totalizando com os dois levantamentos 1225 pesquisas. Para a organização dos resultados foi utilizado o software de tratamento estatístico Sphinx IQ2, que possibilita organizar os dados coletados em gráficos e tabelas, buscando examinar: 


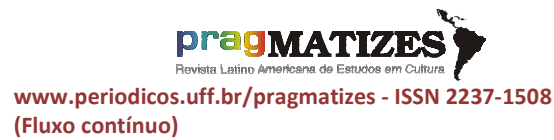

(Fluxo contínuo) $\checkmark$ Quem e quando investigaram?

$\checkmark$ Sobre o que e quem investigaram?

Assim, a organização dos dados deu-se a partir do recorte temporal abarcado pelo balanço de 1987 a 2017 subdividido em três períodos:

$\checkmark$ Período 1: 1987 a 1999

$\checkmark$ Período 2: 2000 a 2009

$\checkmark$ Período 3: 2010 a 2017

Para a determinação dos períodos, foram estabelecidos dois critérios de subdivisão:

O primeiro critério refere-se ao corte temporal no ano 2000 , pois leva em conta o Manifesto "A educação que nós surdos queremos" tornado público em abril de 1999 e demais legislações específicas que foram regulamentadas a partir desse momento. Isso se faz relevante porque a educação de surdos passa a ter alguns de seus aspectos normatizados em decretos e leis de forma independente das demais deficiências (BRASIL, 2002 e 2005).

O segundo corte temporal, em 2010, fez-se devido à mudança ocorrida no Ministério da Educação (MEC) em que a Educação Especial deixa de ser uma atribuição da Secretaria de Educação Especial (Seesp), com a extinção dessa secretaria, e passa à Secretaria de Educação Continuada, Alfabetização, Diversidade e Inclusão - SECADI. Vale ressaltar que essa secretaria já existia sob a denominação de Secretaria de Educação Continuada, Alfabetização e Diversidade (SECAD) e em 2011 ela incorpora em suas funções as ações sobre a inclusão. Tal mudança ocorrida no ministério deflagra uma tensão política e acadêmica que já se processava em anos anteriores e que passa a figurar maior enfrentamento por parte dos profissionais da educação de surdos, que buscam manter as escolas exclusivas (para surdos) diante das ações promovidas pela SECADI, voltadas à inclusão escolar.

Desse modo, os dois recortes temporais foram determinados a partir de dois pontos de inter-relações dos campos político e acadêmico na área específica da educação de surdos e contribuem para a organização dos resultados que são apresentados a seguir.

\section{Quem e quando investigaram?}

Os dados sobre a distribuição da produção em relação à instância 
Tabela 1 - Distribuição da produção por instância administrativa, nos três períodos.

\begin{tabular}{lcccccccc}
\hline \multirow{2}{*}{ Ano } & \multicolumn{2}{c}{$1987 / 1999$} & \multicolumn{2}{c}{$2000 / 2009$} & \multicolumn{2}{c}{$2010 / 2017$} & \multicolumn{2}{c}{ Total } \\
Instância & $\mathrm{N}^{\circ}$ & $\mathrm{M} / \mathrm{A}$ & $\mathrm{N}^{\circ}$ & $\mathrm{M} / \mathrm{A}$ & $\mathrm{N}^{\circ}$ & $\mathrm{M} / \mathrm{A}$ & $\mathrm{N}^{\circ}$ & $\mathrm{M} / \mathrm{A}$ \\
\hline Federal & 49 & 3,8 & 212 & 21,0 & 368 & 46,0 & 629 & 20,3 \\
Privada & 42 & 3,2 & 143 & 14,0 & 144 & 18,0 & 329 & 10,6 \\
Estadual & 24 & 1,9 & 101 & 10,0 & 127 & 15,9 & 252 & 8,13 \\
Municipal & 1 & 0,1 & 3 & 0,3 & 5 & 0,6 & 9 & 0,29 \\
Comunitária & 0 & 0,0 & 0 & 0,0 & 6 & 0,7 & 6 & 0,19 \\
\hline Total & 116 & 9,0 & 459 & 46,0 & 650 & 81,5 & 1225 & 39,5 \\
\hline
\end{tabular}

Fonte: Tabela produzida pela autora a partir de levantamento no Banco de Teses da Coordenação de Aperfeiçoamento de Pessoal de Nível Superior (CAPES). Legenda: M/A corresponde à média anual.

Verifica-se que as pesquisas no campo da educação de surdos aumentaram significativamente nos últimos 31 anos, nas instâncias Federal, Privada e Estadual,com média anual de 9 produções no primeiro período, ultrapassando 81 produções após 17 anos,notando pouca incidência naquelas de instância municipal e comunitária.

Cabe mencionar que a incidência na instância Federal é resultante da disseminação das investigações voltadas à educação de surdos correlacionadas à criação de 18 Universidades Federais e 360 unidades de Institutos Federais de Educação, Ciência e Tecnologia a partir dos anos 2000 (CARREIRA,
2017), além do crescimento exorbitante daquelas de caráter privado nas últimas décadas.

De acordo com o Censo da Educação Superior divulgado pelo Instituto Nacional de Estudos e Pesquisas Educacionais Anísio Teixeira em 2017, a distribuição das instituições de ensino superior no Brasil era de 2.152 Instituições privadas e de 296 públicas, sendo estas distribuídas em 41,9\% estaduais; $36,8 \%$, federais e $21,3 \%$, municipais (BRASIL. INEP, 2017).

Entende-se, portanto, que na educação de surdos as instituições de ensino superior públicas concentram maior incidência de investigações, e a diferença entre as produções oriundas 


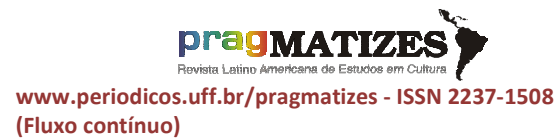

(Fluxo contínuo) dessas instituições fica ainda maior, quando se verifica que o número de IES públicas abrange aproximadamente $14 \%$ do número de instituições privadas.

Os dados referentes à distribuição das pesquisas por instituições de ensino superior (IES) compreende o conjunto de 150 Universidades, Centros Universitários e Institutos Federais distintos. Tendo em vista a complexidade em analisar o rol completo, optou-se em dividir as IES em três blocos, a partir da organização de suas principais diferenças, quais sejam:

$\checkmark$ No primeiro grupo foram organizados os dados das IES que apresentaram produção permanente e relevante durante os 31 anos, o que diz respeito a um conjunto de 10 universidades com maior tradição na área da educação especial, motivo pelo qual será analisado mais detalhadamente, conforme apresenta a tabela 2 .

$\checkmark$ No segundo grupo há a organização dos dados das IES (ANEXO - Grupo 2) que apresentaram produção mais significativa a partir dos anos
2000, reúne um conjunto de 25 IES, principalmente estaduais e federais, contando com pelo menos uma IES de cada região do país.

$\checkmark$ No terceiro grupo foram organizados os dados das IES (ANEXO - Grupo 3) que apresentaram maior dispersão nas produções, pois reúne número expressivo de espaços acadêmicos distintos, em um conjunto de 113 universidades de praticamente todas as regiões do país, compreendendo instâncias estaduais, federais e privadas que possuem reduzida produção na área, com início das publicações principalmente após $\mathrm{o}$ ano de 2010.

Vale destacar duas IES que não se enquadraram nessa organização: UNIFESP e PUCRS, pois apresentaram irregularidade na produção durante os três períodos. Enquanto a produção da UNIFESP é menor nos dois primeiros períodos e mais significativa no terceiro, no caso da PUCRS a produção mais significativa encontra-se somente no primeiro e segundo período. 
A distribuição das produções do

apresentadas na tabela a seguir.

primeiro grupo de IES são

Tabela 2 - Distribuição da produção por IES, nos três períodos.

\begin{tabular}{|c|c|c|c|c|c|c|c|c|}
\hline & & & & & & & & \\
\hline & 1987/1999 & & $2000 / 2009$ & & $2010 / 2017$ & & Total & \\
\hline Instituição & $\mathrm{N}^{\circ}$ & $\mathrm{M} / \mathrm{A}$ & $\mathrm{N}^{\circ}$ & $\mathrm{M} / \mathrm{A}$ & $\mathrm{N}^{\circ}$ & $\mathrm{M} / \mathrm{A}$ & $\mathrm{N}^{\circ}$ & $\mathrm{M} / \mathrm{A}$ \\
\hline PUCSP & 27 & 2,1 & 39 & 3,9 & 18 & 2,3 & 84 & 2,7 \\
\hline USP & 5 & 0,4 & 39 & 3,9 & 24 & 3,0 & 68 & 2,2 \\
\hline UFRGS & 8 & 0,6 & 25 & 2,5 & 24 & 3,0 & 57 & 1,9 \\
\hline UNICAMP & 9 & 0,7 & 25 & 2,5 & 15 & 1,9 & 49 & 1,6 \\
\hline UFSC & 1 & 0,1 & 26 & 2,6 & 20 & 2,5 & 47 & 1,5 \\
\hline UFSCar & 7 & 0,6 & 24 & 2,4 & 16 & 2,0 & 47 & 1,5 \\
\hline UnB & 5 & 0,4 & 19 & 1,9 & 22 & 2,8 & 46 & 1,48 \\
\hline UFSM & 7 & 0,6 & 12 & 1,2 & 14 & 1,8 & 33 & 1,1 \\
\hline UERJ & 9 & 0,7 & 7 & 0,7 & 8 & 1,0 & 24 & 0,8 \\
\hline UFRJ & 5 & 0,4 & 6 & 0,6 & 10 & 1,2 & 21 & 0,7 \\
\hline Outras3 & 33 & 2,5 & 237 & 23,7 & 261 & 32,6 & 749 & 24,0 \\
\hline Total & 116 & 9,0 & 459 & 46,0 & 652 & 81,5 & 1225 & 39,5 \\
\hline
\end{tabular}

Fonte: Tabela produzida pela autora a partir de levantamento no Banco de Teses da Coordenação de Aperfeiçoamento de Pessoal de Nível Superior (CAPES). Legenda: M/A corresponde a média anual.

\footnotetext{
${ }^{3}$ Foram reunidas em "Outras" as Universidades, Centros Universitários e Institutos Federais que tiveram menos de 1 produção na média anual do período total que é abarcado pelo levantamento e compreendem os outros dois blocos de IES caracterizados.
} 


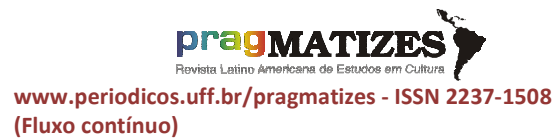

De acordo com os dados apresentados na tabela 2, composta somente pelas dez instituições de ensino superior que mais se destacaram, verifica-se crescimento exponencial de pesquisas na área da educação de surdos, passando de 9 pesquisas anuais, em média, no primeiro período, para 46 e depois 81 , respectivamente, nos períodos subsequentes.

Importante mencionar que, com exceção da PUCSP, todas as demais instituições universitárias são de caráter público. Quatro dessas são paulistas e somam 248 pesquisas em todo o período; duas são gaúchas e somam 90 produções; duas são cariocas com 45; uma catarinense com 47 pesquisas e uma do Distrito Federal com 46.

No entanto, ao estabelecer a média por Unidade de Federação, sem considerar as diferenças entre as respectivas IES, constata-se que: São Paulo possui em média 62 produções por IES; Rio Grande do Sul tem em média 45 por IES, Santa Catarina possui 47; o Distrito Federal 46 e o Rio de Janeiro 45 produções, o que mostra um equilíbrio maior entre a produção por Unidade de Federação.
Em relação ao número de investigações em todo $\mathrm{o}$ período abarcado pelo levantamento, três IES (PUCSP, USP e UFRGS) apresentam produção com maior destaque, reunindo de 57 a 84 investigações, seguidas de um conjunto de IES com incidência muito próxima, ficando entre 46 e 49 produções e três instituições com produção mais baixa, a UFSM com 33, UERJ com 24 e UFRJ com 21 produções no período.

Percebe-se, também, que a maior incidência de pesquisas na PUCSP deve-se ao destaque que tinha no $1^{\circ}$ período, na medida em que a produção das demais IES foi muito baixa. Isto é, a soma das produções das demais IES mais incidentes foi de apenas 56 investigações contra 27 da PUCSP. Em relação ao segundo período (2000/2009), a USP se equiparou a PUCSP e todas as demais experimentaram, percentualmente, incremento superior (com exceção da UFRJ e UERJ), além de, no $3^{\circ}$ período, ter sido superada por quatro IES (USP, UFRGS, UFSC e UnB) e ser a que apresentou queda mais expressiva entre a produção do $2^{\circ}$ período para o último, de 39 para 18 
pesquisas, ou seja, menos da metade da incidência do período anterior.

Assim é possível observar que entre as três mais incidentes, a maior constância da produção parece ser a da UFRGS, com 25 e 24 produções, respectivamente, no $2^{\circ}$ e $3^{\circ}$ períodos, já que a USP, embora em proporção menor que a PUCSP, apresentou queda expressiva nesses dois períodos: de 39 para 24 investigações. A partir dos anos 2000 , verificase significativa disseminação das investigações no âmbito dessa temática, o que não significou somente aumento das produções, como também sua descentralização. Sobretudo, em relação à UnB que é a única, em termos quantitativos, que se destaca fora do eixo Sul-Sudeste.

Os dados da tabela a seguir apresentam as áreas de conhecimento segundo os programas de pósgraduação com maior expressão no conjunto dos dados.

Tabela 3 - Distribuição da produção por área de conhecimento, nos três períodos.

\begin{tabular}{lccccccccc}
\hline \multicolumn{1}{c}{ Ano } & $1987 / 1999$ & \multicolumn{3}{c}{$2000 / 2009$} & \multicolumn{3}{c}{$2010 / 2017$} & \multicolumn{3}{c}{ Total } \\
Área de & $\mathrm{N}^{\circ}$ & $\mathrm{M} / \mathrm{A}$ & $\mathrm{N}^{\circ}$ & $\mathrm{M} / \mathrm{A}$ & $\mathrm{N}^{\circ}$ & $\mathrm{M} / \mathrm{A}$ & $\mathrm{N}^{\circ}$ & $\mathrm{M} / \mathrm{A}$ \\
\hline Conhecimento & 52 & 4,0 & 255 & 25,5 & 350 & 44,0 & 657 & 21,2 \\
\hline Educação & 12 & 0,9 & 87 & 8,7 & 112 & 14,0 & 211 & 6,8 \\
Letras/Linguística/ & 24 & 1,9 & 46 & 4,6 & 40 & 5,0 & 110 & 3,5 \\
Linguagem & 12 & 0,9 & 36 & 3,6 & 24 & 3,0 & 72 & 2,3 \\
Fonoaudiologia/Saúde & 16 & 1,2 & 38 & 3,8 & 121 & 15,0 & 175 & 5,6 \\
Psicologia & 116 & 9,0 & 462 & 46,2 & 651 & 81,0 & 1225 & 39,5 \\
\hline Outras* & & & & & & & \\
\hline Total & & & &
\end{tabular}

Fonte: Tabela produzida pela autora a partir de levantamento no Banco de Teses da Coordenação de Aperfeiçoamento de Pessoal de Nível Superior (CAPES). Legenda: M/A corresponde à média anual. ${ }^{*}$ Compreende as áreas de Enfermagem,Comunicação social, Administração, Artes, Serviço social, Computação, Design e informação e Diversidade e Inclusão.

Com relação às diferentes áreas de conhecimento em que as pesquisas foram desenvolvidas, verifica-se que nos últimos 31 anos a educação (geral) é responsável por 21 pesquisas anuais sobre a educação de surdos, representando mais de $50 \%$ das investigações e sendo mais expressiva do que as áreas da psicologia e fonoaudiologia. 
É possível realizar algumas inferências a partir dos resultados apresentados na tabela 3 , pois percebe-se incidência maior de produções, no primeiro período, entre as áreas da "Educação" e "Fonoaudiologia e Saúde", com 1,9 e 4,0 produções anuais em média, e nas décadas posteriores a área de "Letras, Linguísticas e Linguagem" acaba assumindo posição relevante, com destaque para o número de produções entre os anos de 2010 e 2017, chegando a 14 pesquisas anuais.

Entende-se, portanto, que o crescimento progressivo do interesse nos programas da área de "Letras, Linguística e Linguagem" esteja, de alguma forma, relacionado a pesquisas sobre a língua brasileira de sinais (libras), visto que ela passa a ter reconhecimento oficial a partir da lei 10.436/2002 (BRASIL, 2002) e do decreto 5626/2005 (BRASIL, 2005) que regulamenta o uso e difusão da libras para o acesso à educação e torna obrigatória a formação a respeito dessa língua nos cursos superiores para professores e fonoaudiólogos.

A tabela 4, a seguir, mostra a distribuição dos trabalhos por grau acadêmico.

Tabela 4 - Distribuição da produção por grau acadêmico, nos três períodos.

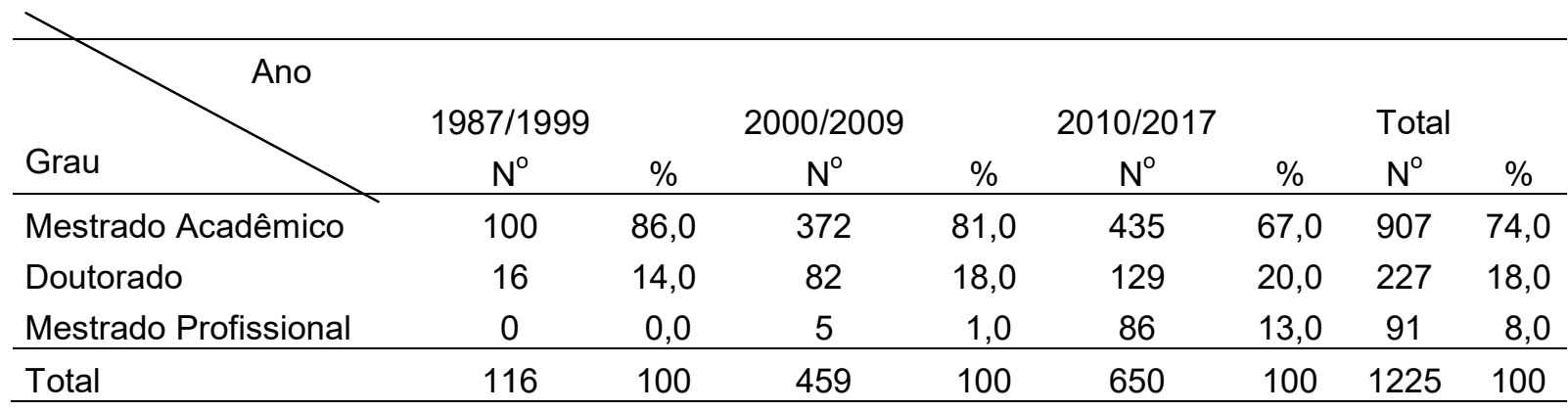

Fonte: Tabela produzida pela autora a partir de levantamento no Banco de Teses da Coordenação de Aperfeiçoamento de Pessoal de Nível Superior (CAPES).

É possível verificar, nos dois primeiros períodos, a predominância de mais de $80 \%$ das publicações em nível de mestrado, isto é, de pesquisas iniciais e pesquisadores iniciantes, como já era de se esperar, pois a duração e dificuldade para atingir tal grau acadêmico é menor do que para o doutorado. No entanto, essa discrepância diminui no último período com o aumento do número deteses de doutorado, possível indicativo das 
políticas de expansão do ensino seu recente reconhecimento, superior nesse período.Sobretudo, justificando assim o aparecimento de verifica-se a consolidação da pesquisa (campo científico) sobre os processos dados sobre esse grau acadêmico somente a partir dos anos 2000.

educacionais de indivíduos com deficiência, conforme já constatado por Bueno (2014), nos quais a educação de surdos contribui de maneira expressiva.

\section{Sobre quem e o que investigaram?}

Os dados sobre o termo utilizado na identificação da deficiência, estão expostos na tabela 5 .

Com relação aos dados sobre o mestrado profissional, vale considerar

Tabela 5 - Distribuição da produção por termo utilizado, nos três períodos.

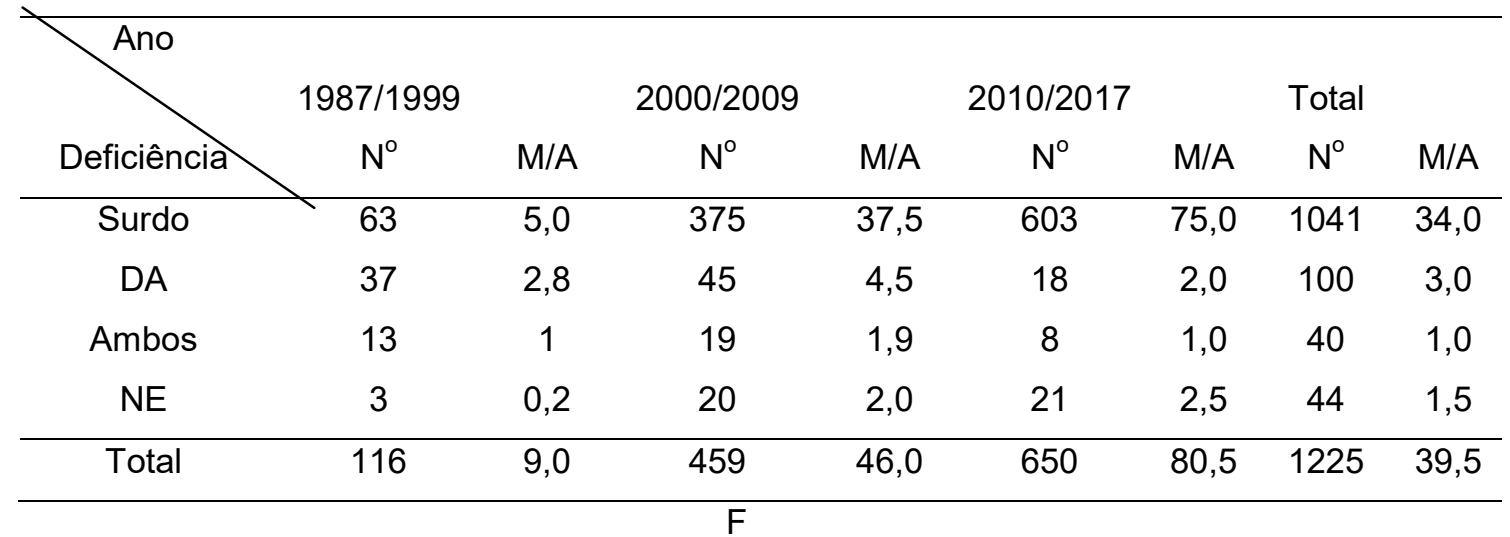

Fonte: Tabela produzida pela autora a partir de levantamento no Banco de Teses da Coordenação de Aperfeiçoamento de Pessoal de Nível Superior (CAPES). Legenda: M/A corresponde a média anual. DA corresponde a Deficiência auditiva. NE corresponde a não especificado.

Observa-se, no primeiro período, que as pesquisas que citam o termo "surdo" ou "deficiente auditivo" apresentam-se em quantidade quase que equiparadas, bem como a utilização de ambos os termos aparecem com relevância nas pesquisas até a primeira década dos anos 2000. Vale ressaltar que a escolha pela utilização por um termo ou outro indica a perspectiva que a investigação tem em relação ao seu objeto de estudo, assim entende-se que entre os anos de 1987 e 1999 coexistiam vertentes que disputavam posição de destaque nesse campo. 
Tal aspecto se modifica no decorrer dos anos subsequentes, pois o termo "surdo" vai sendo incorporado às pesquisas e passa a ser predominante nos anos seguintes (2000-2017), bem como houve decréscimo no número de pesquisas que utilizam as duas expressões.

Com relação a utilização do termo "deficiente auditivo", verifica-se a redução do número de pesquisas que utilizam essa expressão entre os anos de 2010 e 2017, no entanto, o termo se manteve nas pesquisas, sendo unicamente ou em conjunto, como modo de identificação desses sujeitos por todo o período do levantamento.

Sobre isso, o estudo de doutorado de Souza (2018) demonstra que o termo "deficiência" aparece nos artigos da Revista Brasileira de Educação Especial (RBEE), periódico de maior reconhecimento na área, durante todo o período compreendido pelo balanço tendencial realizado pela autora, de 1992 a 2015.Esse achado reitera o que demonstramos dados da tabela 5 , em que parte das pesquisas mais atuais continua utilizando a expressão "deficiência", o que representa mais de $10 \%$ das produções em todo o período.

Fica claro, que a receptividade e ampliação do uso do termo "surdo" ao longo dos últimos 20 anos revela a penetração de certa vertente de estudos no campo da educação especial. Sobretudo, por ser uma receptividade que possui interesses e engajamento político voltados ao reconhecimento dos direitos sociais e educacionais e a visibilidade às dificuldades vivenciadas por essas pessoas.

A tabela a seguir apresenta a distribuição da produção por temática relacionada a língua/linguagem, aspecto elementar nas discussões da área quando se trata de pessoas com surdez ou deficiência auditiva. 
Tabela 6 - Distribuição da produção por língua/linguagem, nos três períodos.

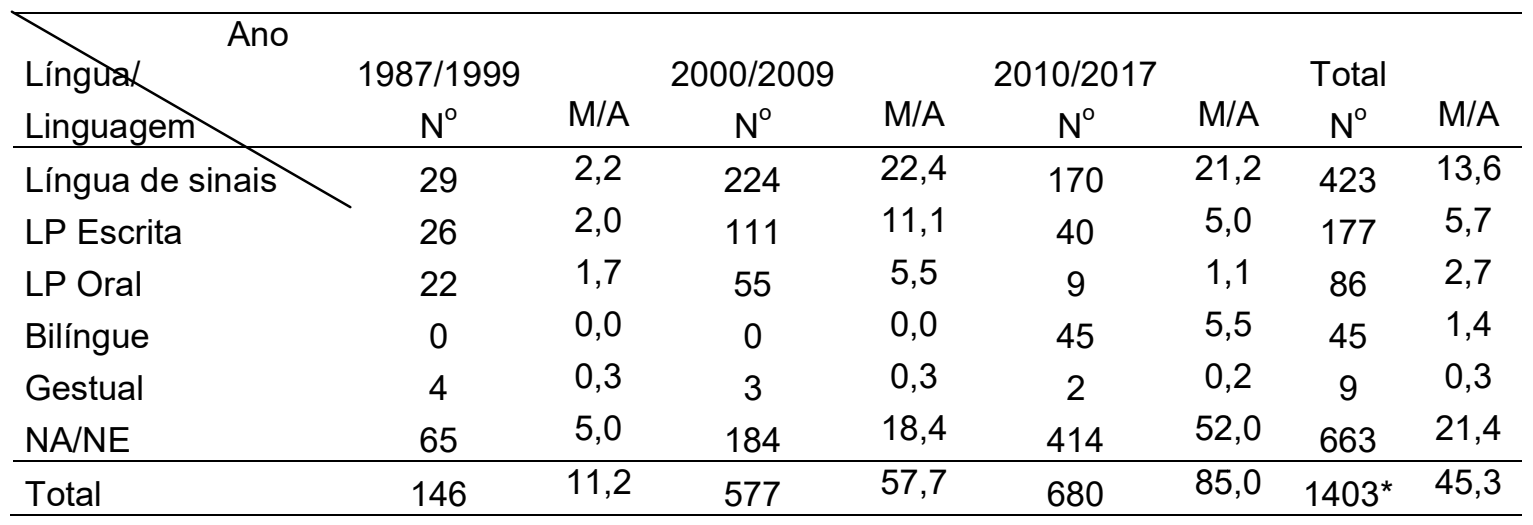

Fonte: Tabela produzida pela autora a partir de levantamento no Banco de Teses da Coordenação de Aperfeiçoamento de Pessoal de Nível Superior (CAPES). Legenda: M/A corresponde à média anual. NA é não aplicável e NE não especificado. LP corresponde à língua portuguesa. *O número total é superior ao número de pesquisas levantadas pois para esse aspecto cabe mais de uma resposta.

De acordo com os dados da tabela 6 , percebe-se que no primeiro período as investigações permeavam proporcionalmente temáticas relacionadas a língua de sinais, língua portuguesa escrita e falada. Mas, nesse mesmo período, na tabela anterior (tabela 3), a maior parte das pesquisas não se mostraram décorrentes da área de "Letras, Linguística e Linguagem", o que leva a entender que essas concentravam outras problemáticas, o que nos remete ao entendimento de Bourdieu (1983, p.89) sobre os interesses específicos que contribuem para definir o campo científico: "[...] não se poderia motivar um filósofo com questões próprias dos geógrafos."

A equiparação no primeiro período entre as três modalidades de língua demonstra a preocupação dos estudiosos com a comunicação, sendo essa oral ou por sinais, mas também com a língua escrita, cujo interesse vai se perdendo ao longo dos períodos posteriores visto que a língua portuguesa nessa modalidade passa a ser considerada, por relevante parte dos pesquisadores, segunda língua da pessoa surda ou língua estrangeira ${ }^{4}$.

Assim, nas décadas subsequentes os estudos sobre a

\footnotetext{
${ }^{4}$ Mais informações sobre Bilinguismo, consultar Quadros (1997).
} 
língua de sinais passam a superar 20 produções anuais, seguidos dos interesses sobre a língua portuguesa escrita ou do estudo de ambas quando se referem ao ensino bilíngue. Este, no caso, somente começa a aparecer a partir de 2010 - em contraste com a queda dos estudos que tratam da língua oral e escrita. Esses indícios sugerem uma reconfiguração do campo científico da educação especial no que se refere ao núcleo que trata da educação de surdos, pois parte dessa produção se retraduz expressivamente em discussões sobre a língua brasileira de sinais.

Não por acaso, autores como Bueno e Ferrari (2013) e Santana e Bergamo (2005, p.578) entendem que há um conjunto de pesquisas da área que acabam por reduzir e negligenciar a complexidade das relações existentes entre linguagem, cultura e a constituição da identidade. É dizer que,

Essa negligência indica que tanto as informações oriundas das pesquisas de campo quanto as discussões provenientes das ciências sociais, [...] passam por uma recepção específica que tem interesse em matizar a questão linguística em detrimento de outras tão importantes quanto, promovendo uma redução arbitrária da complexidade da vida social (SANTANA; BERGAMO, 2005, p. 578).

Em busca de explorar um pouco mais tal discussão, são apresentadas a seguir as temáticas focalizadas nas 1225 pesquisas. 
Tabela 7 - Distribuição das produções por temática investigada, nos três períodos.

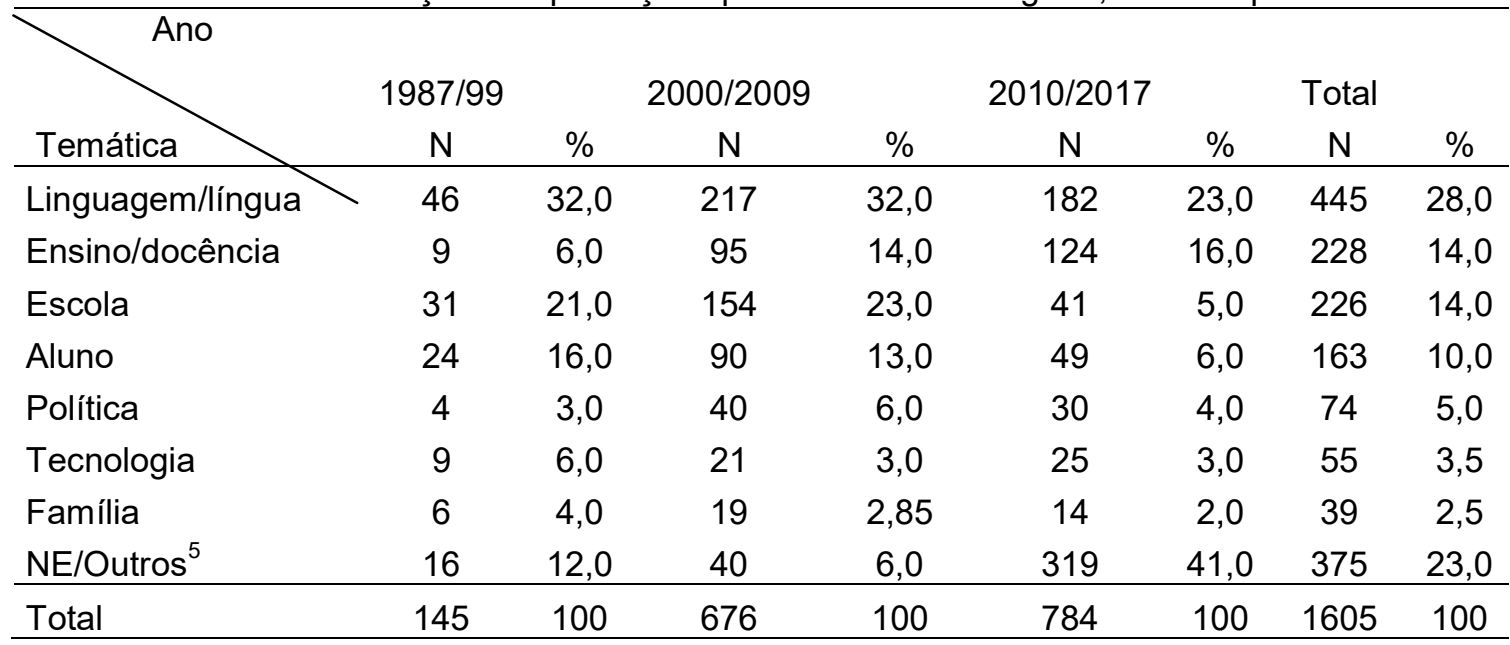

Fonte: Tabela produzida pela autora a partir de levantamento no Banco de Teses da Coordenação de Aperfeiçoamento de Pessoal de Nível Superior (CAPES).

\footnotetext{
${ }^{5}$ Foram selecionadas, neste momento, as temáticas com maior incidência, tendo em vista, a complexidade em analisar o rol completo de campos temáticos, quais sejam: inclusão, escrita, matemática, ensino superior, cultura/arte, clínica/terapêutica, educação especial, saúde, currículo, trabalho, aprendizagem/desempenho, tradutor/intérprete, material didático, representações (surdez), atendimento educacional especializado, movimento social, disciplinas curriculares (física, geografia e química), relações sociais, comunidades surdas, sociedade, trajetórias, interculturalidade, livro, sala de recursos multifuncionais, implante coclear, avaliação, leitura, educação sexual, violência, educação indígena, educação em geral, Instituto Nacional de Educação de Surdos, gênero, escrita de sinais, gestão e raça.
} 


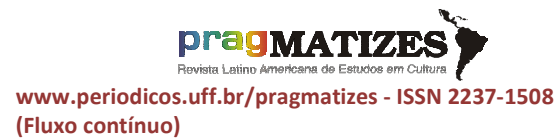
(Fluxo contínuo)
Destaca-se, no primeiro período, as incidências mais elevadas das temáticas voltadas à Linguagem/língua (32\%), à Escola $(21 \%)$ e ao Aluno (16\%),enquanto nos dois períodos posteriores o foco das investigações envolvendo o Ensino e Docência acaba se sobressaindo, chegando a superar a segunda temática mais abordada nos últimos anos.

A preponderância do foco das investigações está no âmbito da Língua e Linguagem nos três períodos destacados, totalizando $28 \%$ das pesquisas, o que equivale ao dobro das duas temáticas seguintes.

Agora, se somarmos os valores das principais temáticas investigadas, que compõem o campo da educação de surdos, três dessas dizem respeito aos aspectos constituidores do processo de escolarização:

$\checkmark$ a escola e suas práticas;

$\checkmark$ o ensino e outros aspectos que permeiam a docência; e

$\checkmark$ o aluno

Sobre isso, constata-se relevante número de pesquisas que voltaram seu foco ao aluno, não com tamanha expressividade como em outras análises (FERREIRA, 1991;
BUENO, 2004 e 2013; SOUZA, 2018) em que o maior número de produções se refere ao alunado da educação especial. De acordo com Bueno (2013), a ênfase que se tem procurado dar aos efeitos que a escolarização e socialização tem ocasionado aos sujeitos, que são a razão de ser da educação especial, explicam essa configuração.

Sobretudo, observa-se a tendência hegemônica dos estudos voltados ao aluno, os quais procuram estabelecer a possível relação entre as condições desse alunado, a organização escolar para atender suas especificidades e a formação e atuação especializada do professor. Isso parece fazer muito sentido aqui, pois o ensino/docência e a escola foram temáticas que alcançaram patamares de destaque, embora, isoladamente, são as discussões sobre a Linguagem/Língua o ponto de preocupação de grande parte das pesquisas desse campo específico.

\section{Algumas considerações}

As análises realizadas evidenciam o que parece ser um traço importante do núcleo da educação de surdos no campo acadêmico da 


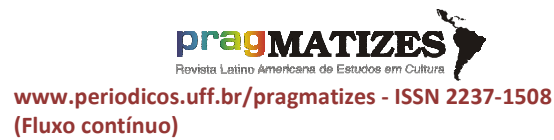

(Fluxo contínuo) educação especial: as principais tendências das pesquisas envolvendo as autorias institucionais demonstram que programas de pós-graduação da área da educação em instituições de ensino superior públicas concentram maior incidência das investigações, e as temáticas e sujeitos sobre as quais se debruçaram permeiam, principalmente, discussões sobre a escola, suas práticas e sujeitos, além dos aspectos da língua e linguagem de sujeitos surdos que utilizam da língua brasileira de sinais.

No entanto, cabe mencionar que o termo "deficiência" continuou sendo utilizado por todo o período, tal como língua portuguesa (falada e escrita), por um conjunto menos expressivo de pesquisas, revelando que vertentes acadêmicas distintas permanecem disputando espaço nesse campo específico.

Por fim, cabe mencionar que este estudo é parte integrante de uma pesquisa maior sobre as "Tendências de investigação no campo da educação de surdos", que por meio de análise das pesquisas sobre a educação desses sujeitos abrange outros dados que não puderam ser incluídos neste texto, devido às limitações exigidas para a composição do dossiê, mas a todo momento busca contribuir para o aprimoramento da pesquisa no campo da educação especial, no sentido de analisar e refletir para além das questões centradas somente nas limitações acarretadas pela surdez, por entender que outros aspectos,como condições econômicas e sociais, raça, idade, gênero e outros, merecem ser apreciados, mas conforme o panorama (inicial) aqui apresentado tais elementos acabam sendo secundarizados.

\section{Referências bibliográficas}

BOTARELLI, G. I. B. O ensinoaprendizagem do português escrito para surdos em pesquisas: análise de resumos acadêmicos (1987-2010). (Mestrado em Educação). Pontifícia Universidade Católica de São Paulo, 2014.

BOURDIEU, P. Coisas ditas. São Paulo: Brasiliense, 2004.

BOURDIEU, P. O campo científico. In: ORTIZ, Ricardo (org.). Pierre Bourdieu: sociologia. São Paulo: Ática, 2013. p. 122-155.

BOURDIEU, P. Questões de sociologia. Rio de Janeiro: Marco Zero, 1983.

BRASIL. Decreto 5.626, de 22 de dezembro de 2005. Regulamenta a Lei 


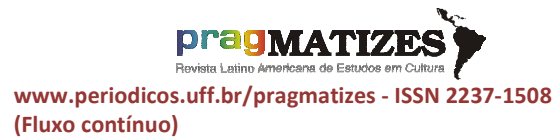

$\mathrm{n}^{0}$ 10.436, de 24 de abril de 2002, que dispõe sobre a Língua Brasileira de Sinais - Libras, e sobre o art. 18 da Lei $\mathrm{n}^{\circ}$ 10.098, de 19 de dezembro de 2000. Disponível em: http://www.planalto.gov.br. Acesso em:15 out. 2015.

BRASIL. Lei n.10.436, de 24 de abril de 2002. Dispõe sobre a Língua Brasileira de Sinais - Libras e dá outras providências. Disponível em: http://www.planalto.gov.br. Acesso em: 16 out. 2015.

BRASIL. MEC. INEP- Instituto Nacional de Estudos e Pesquisas Educacionais Anísio Teixeira. Dados do censo da educação superior: as universidades brasileiras representam $8 \%$ da rede, mas concentram $53 \%$ das matrículas. 2018. Disponível em: http://portal.inep.gov.br/artigo. Acesso em: 10 jan. 2019.

BUENO, J. G. S. A Produção discente do PEPG em Educação: História, Política, Sociedade (PUCSP) no campo dos processos de escolarização, desigualdades sociais e deficiência (2004/2009). In: MELETTI, Silvia Márcia Ferreira; BUENO, José Geraldo Silveira. (orgs.). Políticas públicas, escolarização de alunos com deficiência e a pesquisa educacional. Araraquara: Junqueira \& Marin, 2013. p. 119-138.

BUENO, J. G. S. A pesquisa brasileira sobre educação especial: Balanço tendencial das dissertações e teses brasileiras. In: BUENO, J. G. S.; MUNAKATA, K., CHIOZZINI, D. F. (orgs.). A escola como objeto de estudo: escola, desigualdades, diversidades. Araraquara: Junqueira \& Marin, 2014, p. 211-244.
BUENO, J. G. S. O aluno como foco de investigações sobre a escola: tendências das dissertações e teses defendidas nos programas de pósgraduação em educação - 1981/1998. In: Encontro Nacional de Didática e Prática de Ensino, 12, 2004, Anais[...]. Curitiba: ENDIPE, 2004. 1 CD-ROM.

BUENO, J. G. S; FERRARI, C. C. Contrapontos socioeducacionais da surdez: para além da marca da deficiência. Cadernos tramas da memória, Fortaleza,vol. 1, n. 3, 2013, p. 41-73.

BUENO, J. G. S. Surdez, linguagem e cultura. Cadernos CEDES [online]. Campinas, vol. 19, n. 46, p. 41-56, $1998 . \quad$ Disponível em: http://www.scielo.br/scielo.php?script= sci_arttext\&pid=S0101-

32621998000300005\&ln

$\mathrm{g}=\mathrm{pt \& nrm}=$ iso. Acesso em: 21 set. 2015.

CARREIRA, Denise. Igualdade e Diferenças nas Políticas Educacionais: a agenda das diversidades nos governos Lula e Dilma. São Paulo: Ação Educativa, 2017.

CUKIERKORN, M. M. DE O. $A$ escolaridade especial do deficiente auditivo: estudo crítico sobre os procedimentos didáticos especiais. (Mestrado em Educação). Pontifícia Universidade Católica de São Paulo, 1996.

CUKIERKORN, M. M. DE O. As trajetórias escolares de deficientes auditivos na rede pública municipal de São Paulo. (Doutorado em Educação). Pontifícia Universidade Católica de São Paulo, 2005. 
DANTAS. M. M. D. Práticas cotidianas de ensino da língua escrita em classe especial para surdos. (Mestrado em Educação). Pontifícia Universidade Católica de São Paulo, 2006.

FERRARI, C. C. Os agrupamentos espontâneos de jovens e adultos surdos: um estudo sobre suas trajetórias e composição. (Mestrado em Educação) - Pontifícia Universidade Católica de São Paulo, São Paulo, 2010.

FERRARI, C. C. Surdez, cultura e identidade: as trajetórias sociais na construção das identidades de agentes surdos. (Doutorado em Educação). Pontifícia Universidade Católica de São Paulo, 2017.

FERREIRA, J. R. Pesquisa no contexto da política em Educação Especial. In: Seminário Brasileiro de Pesquisa em Educação Especial, 2, 1991, Rio de Janeiro. Anais[...]. Rio de Janeiro: UERJ, v. 1, 1991. p. 8-11.

LIMA, P. E. A. de. $O$ acesso e permanência de indivíduos surdos ao ensino superior. (Mestrado em Educação). Pontifícia Universidade Católica de São Paulo, 2012.
QUADROS, R. M. de. Educação de surdos: a aquisição da linguagem. Porto Alegre: Artmed, 1997.

SANTANA, A. P. BERGAMO, A. Cultura e Identidade Surdas: encruzilhada de lutas sociais e teóricas. Educação \& Sociedade, Campinas, v.26, n.91, p.565-582, 2005.

SILVA, M. J. dos S. Meio social e surdez: um estudo sobre trajetória sócio-educacional no município de Tocantinópolis - TO. (Mestrado em Educação). Pontifícia Universidade Católica de São Paulo, 2011.

SKLIAR, C. B. Os estudos surdos em educação: problematizando a normalidade. In: SKLIAR, C. B. (org.). A surdez:um olhar sobre as diferenças. Porto Alegre: Mediação, 1998. p. 2950.

SOUZA, S. B de. Excepcionalidade, deficiência ou necessidades educacionais especiais: o aluno como constituinte do campo da educação especial. (Doutorado em Educação). Pontifícia Universidade Católica de São Paulo, 2018. 
FERRARI, Carla Cazelato. Panorama das hierarquias no campo da educação de deficientes auditivos e surdos no período de 1987-2017. PragMATIZES - Revista Latino-Americana de Estudos em Cultura, Niterói/RJ, Ano 12, n. 22, p. 641-662, mar. 2022.
pragMATIZES

www.periodicos.uff.br/pragmatizes - ISSN 2237-1508 (Fluxo contínuo)

\section{ANEXO -IES}

\begin{tabular}{|l|l|}
\hline Sigla & IES - Grupo 2 \\
\hline UFF & Universidade Federal Fluminense \\
\hline UNESP & Universidade Estadual Paulista \\
\hline UFES & Universidade Federal do Espírito Santo \\
\hline UEM & Universidade Estadual de Maringá \\
\hline UFC & Universidade Federal do Ceará \\
\hline UFPA & Universidade Federal do Pará \\
\hline UFPB & Universidade Federal da Paraíba \\
\hline UFMG & Universidade Federal de Minas Gerais \\
\hline UTP & Universidade Tuiuti do Paraná \\
\hline UFBA & Universidade Federal da Bahia \\
\hline UFPR & Universidade Federal do Paraná \\
\hline UFU & Universidade Federal de Uberlândia \\
\hline UNIMEP & Universidade Metodista de Piracicaba \\
\hline PUCRJ & Pontifícia Universidade Católica do RJ \\
\hline UFPE & Universidade Federal de Pernambuco \\
\hline UFPEL & Universidade Federal de Pelotas \\
\hline UPM & Universidade Presbiteriana Mackenzie \\
\hline UFG & Universidade Federal de Goiás \\
\hline UNISINOS & Universidade do Vale do Rio dos Sinos \\
\hline UFAM & Universidade Federal do Amazonas \\
\hline CUML & Centro Universitário Moura Lacerda \\
\hline UFMS & Universidade Federal de Mato Grosso do \\
\hline UFMT & Universidade Federal de Mato Grosso \\
\hline UFS & Universidade Federal de Sergipe \\
\hline UNIR & Universidade Federal de Rondônia \\
\hline
\end{tabular}

\begin{tabular}{|l|l|}
\hline Sigla & IES - Grupo 3 \\
\hline UMESP & Universidade Metodista de São Paulo \\
\hline UNICAP & Universidade Católica de Pernambuco \\
\hline UFRN & $\begin{array}{l}\text { Universidade Federal do Rio Grande do } \\
\text { Norte }\end{array}$ \\
\hline UCB & Universidade Católica de Brasília \\
\hline UECE & Universidade Estadual do Ceará \\
\hline UEL & Universidade Estadual de Londrina \\
\hline ULBRA & Universidade Luterana do Brasil \\
\hline UNIJUI & Universidade Regional do Estado do RGS \\
\hline UVA & Universidade Veiga de Almeida \\
\hline IFG & Universidade Federal de Goiás \\
\hline UEPA & Universidade do Estado do Pará \\
\hline UESB & Universidade Estadual do Sudoeste da \\
\hline
\end{tabular}

\begin{tabular}{|c|c|}
\hline & Bahia \\
\hline UFAL & Universidade Federal de Alagoas \\
\hline UFGD & Universidade Federal da Grande Dourados \\
\hline UFJF & Universidade Federal de Juiz de Fora \\
\hline UNESA & Universidade Estácio de Sá \\
\hline PUCMG & Pontifícia Universidade Católica de MG \\
\hline USF & Universidade São Francisco \\
\hline CUMIPA & Centro Universitário Metodista IPA \\
\hline IFSP & Instituto Federal São Paulo \\
\hline UCPEL & Universidade Católica de Pelotas \\
\hline UERN & $\begin{array}{l}\text { Universidade do Estado do Rio Grande do } \\
\text { Norte }\end{array}$ \\
\hline UFOP & Universidade Federal de Ouro Preto \\
\hline UNIGRANRIO & Universidade do Grande Rio \\
\hline UNILASALLE & Universidade La Salle \\
\hline UNITAU & Universidade de Taubaté \\
\hline UPF & Universidade de Passo Fundo \\
\hline FURG & Universidade Federal do Rio Grande \\
\hline PUCCAMP & $\begin{array}{l}\text { Pontifícia Universidade Católica de } \\
\text { Campinas }\end{array}$ \\
\hline PUCGO & Pontifícia Universidade Católica de Goiás \\
\hline UCDB & Universidade Católica Dom Bosco \\
\hline UDESC & Universidade do Estado de Santa Catarina \\
\hline UEA & Universidade do Estado do Amazonas \\
\hline UEFS & Universidade Estadual de Feira de Santana \\
\hline UEMS & $\begin{array}{l}\text { Universidade Estadual de Mato Grosso do } \\
\text { Sul }\end{array}$ \\
\hline UFPI & Universidade Federal do Piauí \\
\hline UNEB & Universidade do Estado da Bahia \\
\hline UNIAN & Universidade Anhanguera \\
\hline UNIRIO & Universidade Federal do Estado do RJ \\
\hline $\begin{array}{l}\text { Anhembi } \\
\text { Morumbi }\end{array}$ & Universidade AnhembiMorumbi \\
\hline CEFETRJ & $\begin{array}{l}\text { Centro Federal de Educação Tecnológica } \\
\text { do RJ }\end{array}$ \\
\hline CUM & Centro Universitário Metropolitano \\
\hline FURB & Universidade Regional de Blumenau \\
\hline FVC & Faculdade Vale do Cricaré \\
\hline UEG & Universidade Estadual de Goiás \\
\hline UEMG & Universidade do Estado de Minas Gerais \\
\hline UEPB & Universidade Estadual da Paraíba \\
\hline UERR & Universidade Estadual de Roraima \\
\hline UFAC & Universidade Federal do Acre \\
\hline UFRRJ & Universidade Federal Rural do RJ \\
\hline
\end{tabular}


FERRARI, Carla Cazelato. Panorama das hierarquias no campo da educação de deficientes auditivos e surdos no período de 1987-2017. PragMATIZES - Revista Latino-Americana de Estudos em Cultura, Niterói/RJ, Ano 12, n. 22, p. 641-662, mar. 2022.

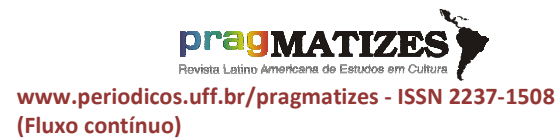
(Fluxo contínuo)

\begin{tabular}{|c|c|}
\hline UFTM & Universidade Federal do Triângulo Mineiro \\
\hline UFV & Universidade Federal de Viçosa \\
\hline UFVJM & $\begin{array}{l}\text { Universidade Federal dos Vales do } \\
\text { Jequitinhonha }\end{array}$ \\
\hline UMC & Universidade de Mogi das Cruzes \\
\hline UNAMA & Universidade da Amazônia \\
\hline UNIFAP & Universidade Federal do Amapá \\
\hline UNIFEI & Universidade Federal de Itajubá \\
\hline UNIFOA & Centro Universitário de Volta Redonda \\
\hline UNIFOR & Universidade de Fortaleza \\
\hline UNIMES & Universidade Metropolitana de Santos \\
\hline UNIMONTES & Centro Universitário Monte Serrat \\
\hline UNINOVE & Centro Universitário Nove de Julho \\
\hline UNIOESTE & Universidade Estadual do Oeste do Paraná \\
\hline UNIPLI & Centro Universitário Plínio Leite \\
\hline UNISAL & $\begin{array}{l}\text { Centro Universitário Salesiano de São } \\
\text { Paulo }\end{array}$ \\
\hline UNISC & Universidade de Santa Cruz do Sul \\
\hline UNISO & Universidade de Sorocaba \\
\hline UNIT & Centro Universitário Tiradentes \\
\hline UNIUBE & Universidade de Uberaba \\
\hline UNIVALI & Universidade do Vale do Itajaí \\
\hline UNIVAS & Universidade do Vale do Sapucaí \\
\hline URI & $\begin{array}{l}\text { Universidade Regional Integrada do Alto } \\
\text { Uruguai e Das Missões }\end{array}$ \\
\hline USM & Universidade São Marcos \\
\hline UTFPR & $\begin{array}{l}\text { Universidade Tecnológica Federal do } \\
\text { Paraná }\end{array}$ \\
\hline CEFETMG & $\begin{array}{l}\text { Centro Federal de Educação Tecnológica } \\
\text { de MG }\end{array}$ \\
\hline CESJF & Centro de Ensino Superior de Juiz de Fora \\
\hline FUVATES & $\begin{array}{l}\text { Fundação Vale do Taquari de Ed. e } \\
\text { Desenvolvimento Social }\end{array}$ \\
\hline HOSPHEL & $\begin{array}{l}\text { Centro Universitário de Ciências Biológicas } \\
\text { e Saúde - Hospital Heliópolis }\end{array}$ \\
\hline IES & $\begin{array}{l}\text { Instituto de Ensino Superior da Grande } \\
\text { Florianópolis }\end{array}$ \\
\hline IFAM & Instituto Federal do Amazonas \\
\hline IFES & Instituto Federal do Espírito Santo \\
\hline
\end{tabular}

\begin{tabular}{|c|c|}
\hline IFRJ & Instituto Federal do Rio de Janeiro \\
\hline IFSUL & Instituto Federal Sul-rio-grandense \\
\hline ITA & Instituto Tecnológico de Aeronáutica \\
\hline PUCPR & Pontifícia Universidade Católica do Paraná \\
\hline UBC & Universidade Braz Cubas \\
\hline UCS & Universidade de Caxias do Sul \\
\hline UENF & $\begin{array}{l}\text { Universidade Estadual do Norte } \\
\text { Fluminense }\end{array}$ \\
\hline UEPG & Universidade Estadual de Ponta Grossa \\
\hline UESC & Universidade Estadual de Santa Cruz \\
\hline UFCG & Universidade Federal de Campina Grande \\
\hline UFERSA & Universidade Federal Rural do Semi-árido \\
\hline UFLA & Universidade Federal de Lavras \\
\hline UFMA & Universidade Federal do Maranhão \\
\hline UFRR & Universidade Federal de Roraima \\
\hline UFSJ & Universidade Federal de São João Del-Rei \\
\hline UGF & Universidade Gama Filho \\
\hline UNESC & Universidade do Extremo Sul Catarinense \\
\hline UNESPAR & Universidade Estadual do Paraná \\
\hline UNIBAN & Universidade Bandeirante de São Paulo \\
\hline UNICID & Universidade Cidade de São Paulo \\
\hline UNINCOR & Universidade Vale Do Rio Verde \\
\hline UNIFRA & Universidade Franciscana \\
\hline UNEMAT & Universidade do Estado de Mato Grosso \\
\hline UNINTER & Centro Universitário Internacional \\
\hline UNIPLAC & Universidade do Planalto Catarinense \\
\hline UNISANTOS & Universidade Católica de Santos \\
\hline UNIVALE & Universidade Vale do Rio Doce \\
\hline UNIVATES & Universidade do Vale do Taquari \\
\hline UNIVERSO & Universidade Salgado de Oliveira \\
\hline UNOCHAPECÓ & $\begin{array}{l}\text { Universidade Comunitária da Região de } \\
\text { Chapecó }\end{array}$ \\
\hline UNOESTE & Universidade do Oeste Paulista \\
\hline USU & Universidade Santa Úrsula \\
\hline
\end{tabular}

